\title{
Experimental Evidence of Self-Regulation of Fluctuations by Time-Varying Flows
}

\author{
M. G. Shats* and W. M. Solomon \\ Plasma Research Laboratory, Australian National University, Canberra ACT 0200 Australia
}

(Received 17 July 2001; published 8 January 2002)

\begin{abstract}
We report the first extended experimental results indicating that radially localized time-varying potential structures, which possess many of the characteristics of zonal flows, are generated by strong fluctuations. Experiments performed in the $\mathrm{H}-1$ heliac show that these poloidally symmetric flows are nonlinearly coupled to other fluctuations and are responsible for significant modifications in fluctuations and in the fluctuation-driven transport.
\end{abstract}

DOI: $10.1103 /$ PhysRevLett.88.045001

It is known that plasma turbulence can generate flows (for review, see [1]). Since shear flows affect turbulence through the shear decorrelation mechanism [2], it is expected that the flow/turbulence dynamics controls the turbulence level and, as a result, affects the turbulent transport. Considerable theoretical effort is concentrated on the physics of zonal flows [3,4]. Zonal flows (ZF) are anisotropic potential structures, which are toroidally and poloidally symmetric (poloidal and parallel wave numbers $k_{\theta} \approx 0, k_{\|} \approx 0$ ) and are radially localized (radial wave number $k_{r} \neq 0$ ) [4]. Such structures are generated by the turbulence and can be described as low-frequency $\tilde{\mathbf{E}} \times \mathbf{B}$ shear flows. The theoretical effort (e.g., Refs. [5-7]) supported by simulations (e.g., Refs. [8-10]) has resulted in a detailed description of the generation of zonal flows. Several suggestions on the manifestation of zonal flows in experiments have been proposed (e.g., Refs. [11,12]) and several observations indirectly identifying zonal flows have been reported [12-15]. Further progress in this direction requires more feedback from experiments. Such experiments are not easy to perform in view of apparent difficulties with multipoint time- and space-resolved measurements of the ZF characteristics in the inner regions of high-temperature plasmas. In this Letter we present experimental results from a low-temperature toroidal plasma, where a large number (14) of probes have been used to characterize $\tilde{\mathbf{E}} \times \mathbf{B}$ flows generated by the nonlinearity of the fluctuations of the plasma parameters.

$\tilde{\mathbf{E}} \times \mathbf{B}$ shear flows can be generated due to the turbulent Reynolds stress [16] or through other mechanisms in which fluctuations drive radial currents and therefore contribute to the poloidal momentum balance equation:

$$
\partial V_{\theta} / \partial t=-\partial / \partial r\left(\tilde{V}_{r} \tilde{V}_{\theta}\right)-J_{r} B_{\phi} /\left(m_{i} n\right)-\mu V_{\theta} .
$$

Here $V_{\theta}$ and $V_{r}$ are poloidal and radial flow velocities, $B_{\phi}$ is the toroidal magnetic field and $\mu$ is the damping rate of the mean flow. A radial current $J_{r}$ can result from the nonambipolarity of the fluctuation-driven transport, $J_{r}^{\mathrm{fl}}=$ $e\left\langle\tilde{n}\left(\tilde{V}_{\text {er }}-\tilde{V}_{\text {ir }}\right)\right\rangle$, if the electron and ion radial fluctuating velocities are different, $\tilde{V}_{\text {er }} \neq \tilde{V}_{\text {ir }}$, for example, due to the finite ion Larmor radius effect. This mechanism is known to be capable of generating zonal flows [17]. It has recently been reported [18] that the fluctuations in the $\mathrm{H}-1$ heliac
PACS numbers: 52.35.Ra, 52.35.Mw, 52.55.Hc

generate radial currents so that the 2nd term in the righthand side of Eq. (1) appears to be larger than the turbulent Reynolds stress (1st term). Thus the nonambipolarity of the fluctuation-driven particle fluxes could also lead to the generation of a time-varying radial electric field.

The ZF generation mechanism has been considered as a mode-coupling problem in which shorter-scale turbulent velocity fluctuations transfer their energy nonlinearly to larger-scale potential structures $[12,14,15]$. The energy is transferred via 3-wave interactions. In the presence of broadband turbulence the process is amplified due to the inverse cascading. Although the interaction between zonal flows and the broadband spectra of their parent waves is an important feature of a ZF-turbulence system, the existence of a broadband turbulence is not necessary for the ZF formation. Following the formalism of the work [14] it is easy to show that, in the case when the nonambipolar turbulent transport dominates over the turbulent Reynolds stress in Eq. (1), the energy transfer between the smaller-scale fluctuations and larger-scale shear flows is proportional to $\sum_{k=k_{1}+k_{2}} \operatorname{Re}\left[\left\langle\tilde{E}_{r}^{*}(k) \tilde{n}\left(k_{1}\right) \tilde{E}_{\theta}\left(k_{2}\right)\right\rangle\right]$, where $\tilde{E}_{r}^{*}(k)$ and $\tilde{E}_{\theta}\left(k_{2}\right)$ are fluctuations in the radial and poloidal electric fields, respectively (asterisk denotes the complex conjugate), and $\tilde{n}\left(k_{1}\right)$ represents density fluctuations. The wave numbers satisfy the selection rule for 3-wave processes, $k=k_{1}+k_{2}$. Thus, changes in the degree of nonlinear coupling between the three fluctuating quantities should be reflected in the real part of the bispectrum $B_{k}\left(k_{1}, k_{2}\right)=\sum_{k=k_{1}+k_{2}}\left\langle\tilde{E}_{r}^{*}(k) \tilde{n}\left(k_{1}\right) \tilde{E}_{\theta}\left(k_{2}\right)\right\rangle$, which can be determined in experiments. Among other features of ZF that can be detected experimentally are their poloidal symmetry and the radial localization.

In this Letter we present experimental evidence of the generation of the ZF-like potential structures via 3-wave interactions of nonlinearly coupled waves. It is shown that the enrichment of the fluctuation spectrum coincides with an increase in the non-linear mode coupling and results in the formation of the ZF-like structures. It is demonstrated experimentally for the first time that these nonlinearly generated potential structures (i) have a radial wave number much larger than the poloidal wave number, (ii) are radially localized, (iii) do not generate any particle transport themselves, but (iv) modulate transport driven by their 
parent waves. All these properties predicted for zonal flows driven by drift wave turbulence are observed in the low-temperature plasma experiments reported here.

Experiments have been performed on the $\mathrm{H}-1$ heliac [19], a 3-field period helical axis stellarator which has a major radius of $R=1 \mathrm{~m}$ and a mean minor radius of about $\langle a\rangle \approx 0.2 \mathrm{~m}$. Parameters of the plasma produced by $\sim 60 \mathrm{~kW}$ of radio-frequency waves at $7 \mathrm{MHz}$ were as follows: $n_{e} \sim 1 \times 10^{18} \mathrm{~m}^{-3}, T_{e} \sim 10 \mathrm{eV}, T_{i} \sim 40 \mathrm{eV}$ [20]. In the reported experiments, plasma is produced in argon at filling pressures $(1-3) \times 10^{-5}$ torr. Several combinations of triple probes [21] are used to characterize plasma parameters. Triple probes provide a measurement of the ion saturation current $I_{s}$, electron temperature $T_{e}$, and the plasma potential $\phi_{p}$. The poloidal electric field is estimated from two poloidally separated triple probes as $\tilde{E}_{\theta}=\left(\tilde{\phi}_{p 2}-\tilde{\phi}_{p 1}\right) / \Delta y$. Simultaneously we measure the radial electric field using two triple probes, which are separated radially: $E_{r}=\left(\phi_{r 1}-\phi_{r 2}\right) / \Delta r$. All probes are radially and poloidally aligned using the electron beam mapping technique in the stellarator vacuum magnetic field.

Strong fluctuations in the electron density and electrostatic potential, identified as unstable resistive pressuregradient-driven modes [22], are typical for this plasma. These fluctuations are suppressed when the time-average radial electric field shear exceeds some critical $E_{r}^{\prime}[22,23]$. The suppression of fluctuations leads to an improvement in the particle confinement, characterized by an increased plasma density, more peaked ion pressure profiles and a stronger $E_{r}$ shear $[18,22]$, somewhat similar to $H$ mode in other toroidal experiments. Under some conditions fluctuations may recover in the improved confinement mode $[22,24]$. In this paper we study fluctuations in the radial electric field $\tilde{E}_{r}$, poloidal electric field $\tilde{E}_{\theta}$ and in the electron density $\tilde{n}_{e}$ in both low $(L)$ and high $(H)$ confinement modes.

First we consider the $L$-mode fluctuations. Figure 1(a) shows radial profiles of the root-mean-square level of the fluctuations in the ion saturation current $\operatorname{rms}\left(\tilde{I}_{s}\right) \propto$ $\operatorname{rms}\left(\tilde{n}_{e}\right), 1(\mathrm{~b})$ relative level (normalized to the mean radial electric field) of the strongest harmonic in $\tilde{E}_{r}$ fluctuations, $\operatorname{rms}\left(\tilde{E}_{r}\right) /\left\langle E_{r}\right\rangle$, and 1(c) the poloidal wave number of the plasma potential fluctuations derived from the phase shift measured using two poloidally separated triple probes. The $\tilde{E}_{r}$ fluctuations are localized in a relatively narrow radial region of $r / a=(0.45-0.75)$ in the plasma. Poloidal wave numbers of the plasma potential fluctuations [Fig. 1(c)] are close to zero inside this radial region. Shown in Figs. 2(a) and 2(b) are the frequency spectra of the fluctuations of the radial and poloidal electric fields. The $\tilde{E}_{r}$ fluctuation level is higher than that of $\tilde{E}_{\theta}$ and the frequencies of the strongest $\tilde{E}_{r}$ and $\tilde{E}_{\theta}$ harmonics do not coincide. The maximum in $\tilde{E}_{r}$ corresponds to the wave number in the plasma potential $k_{r} \gg k_{\theta} \approx 0$, while all other components in the frequency spectra of the plasma potential have $k_{\theta} \geq k_{r}$. To evaluate the degree of the
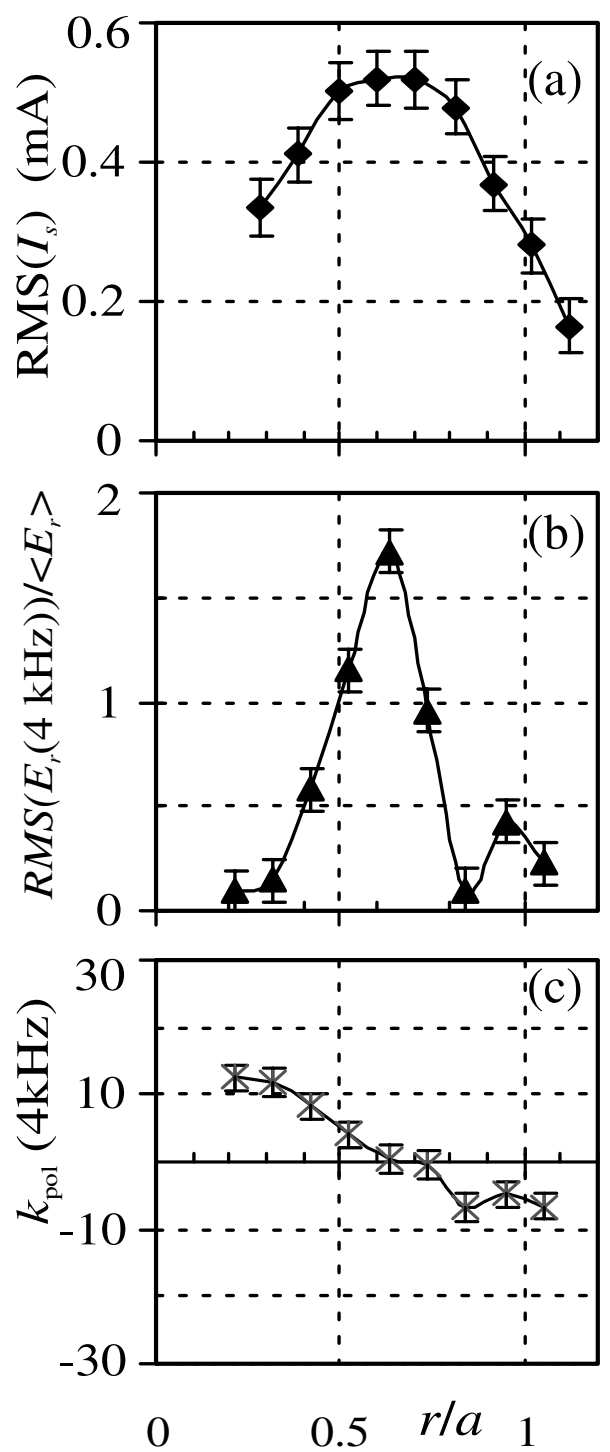

FIG. 1. Radial profiles of the ion saturation current $\tilde{I}_{s} \sim$ $\tilde{n}_{e} \sqrt{T_{e}+T_{i}}$ (a), normalized radial electric field fluctuations $\operatorname{rms}\left(\tilde{E}_{r}\right) /\left\langle E_{r}\right\rangle$ at $f=4.2 \mathrm{kHz}(\mathrm{b})$, and poloidal wave number at $f=4.2 \mathrm{kHz}$ (c).

3-wave coupling between $\tilde{E}_{r}, \tilde{E}_{\theta}$, and $\tilde{n}_{e}$ we compute the squared crossed bicoherence (normalized bispectrum), which is independent of the fluctuation amplitude:

$$
b_{f}^{2}\left(f_{1}, f_{2}\right)=\frac{\left|\left\langle E_{r}(f)^{*} n\left(f_{1}\right) E_{\theta}\left(f_{2}\right)\right\rangle\right|^{2}}{\left\langle E_{r}(f) E_{r}(f)^{*}\right\rangle\left\langle\left|n\left(f_{1}\right) E_{\theta}\left(f_{2}\right)\right|^{2}\right\rangle} .
$$

Here predictions for the $k$ domain are transformed to the frequency domain (similar to $[14,15]$ ), so that the phase-coherent 3-wave interaction obeys the frequency selection rule, $f=f_{1}+f_{2}$. We also compute the summed cross-bicoherence (SCB), $\quad \operatorname{SCB}(f)=$ $\sum_{f=f_{1}+f_{2}} b_{f}^{2}\left(f_{1}, f_{2}\right)$, which gives a measure of the coherent 3-wave coupling for all frequencies satisfying $f=f_{1} \pm f_{2}$. The $b_{f}^{2}\left(f_{1}, f_{2}\right)$ contour plot shown in Fig. 2(c) indicates that all harmonics in the frequency spectrum of Figs. 2(a) and 2(b) are nonlinearly 

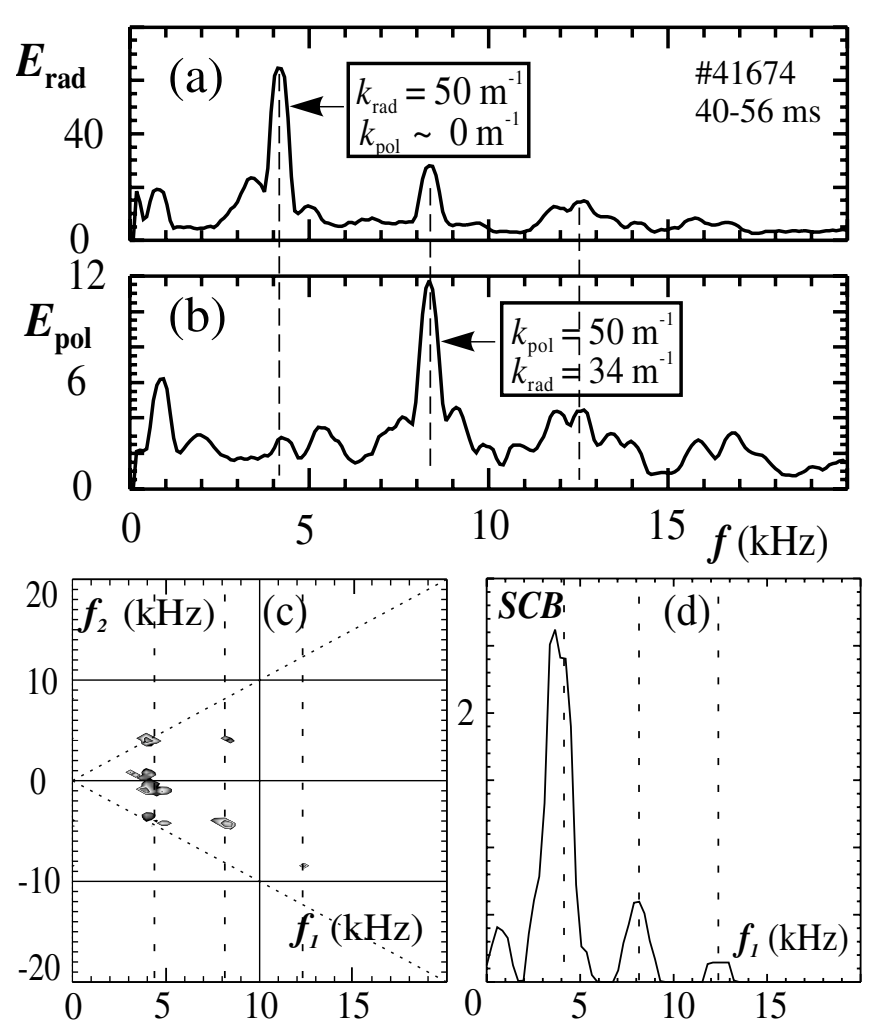

FIG. 2. Frequency spectra of the radial (a) and poloidal (b) electric field fluctuations, contour plot of the crossed bicoherence, [Eq. (2)] (c), and the summed cross bicoherence (d) for the fluctuation data of Fig. 1 measured at $r / a=0.67$.

coupled $\left(f_{1}=0.8, \quad F_{r}=4.2, \quad f_{2}=8.4, \quad f_{3}=11.8\right.$, and $f_{4}=12.6 \mathrm{kHz}$ ) and obey the frequency selection rules such that $f_{1}=f_{4}-f_{3}$ and $F_{r}=f_{4}-f_{2}$. A summed cross bicoherence [Fig. 2(d)] has a maximum at $F_{r} \approx 4 \mathrm{kHz}$, which suggests that the strongest $\tilde{E}_{r}\left(F_{r}\right)$ harmonic [also characterized by the zero poloidal wave number $k_{\theta}\left(F_{r}\right) \approx 0$ [Fig. 2(a)] and a distinct radial localization [Fig. 1(b)] ] has the strongest nonlinear coupling with other fluctuation harmonics. Unlike other harmonics, which have finite $k_{\theta}$, this structure does not generate any significant particle transport because of its poloidal symmetry, $\Gamma_{\mathrm{fl} 1}\left(F_{r}\right)=k_{\theta}\left(F_{r}\right)\left\langle\tilde{n}\left(F_{r}\right) \tilde{\phi}_{p}\left(F_{r}\right)\right\rangle / B_{t} \approx 0$. All these characteristics are indicative of the ZF-like structure in the low confinement mode.

Now we consider fluctuations that develop in the improved confinement mode $[22,24]$ after the suppression of the fluctuations described above. An interesting feature of the fluctuations in $H$ mode is that the developing instability can often be observed at the early stage when the initially monochromatic frequency spectrum gradually becomes rich and the number of harmonics increases with time, as illustrated in Fig. 3. The fluctuation timefrequency dynamics is analyzed using a continuous wavelet technique (the Morlet wavelet) [25-27]. Shortly after the onset of fluctuations $(t=44-54 \mathrm{~ms})$ the $f=11.2 \mathrm{kHz}$ harmonic dominates the frequency spectra of the fluctuations in the electron density [Fig. 3(a)],
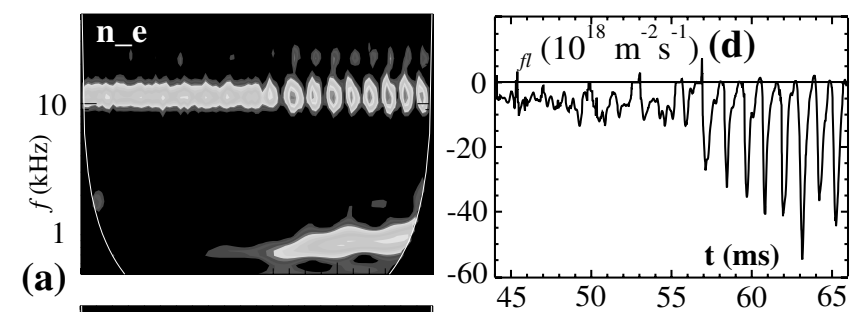

(a)
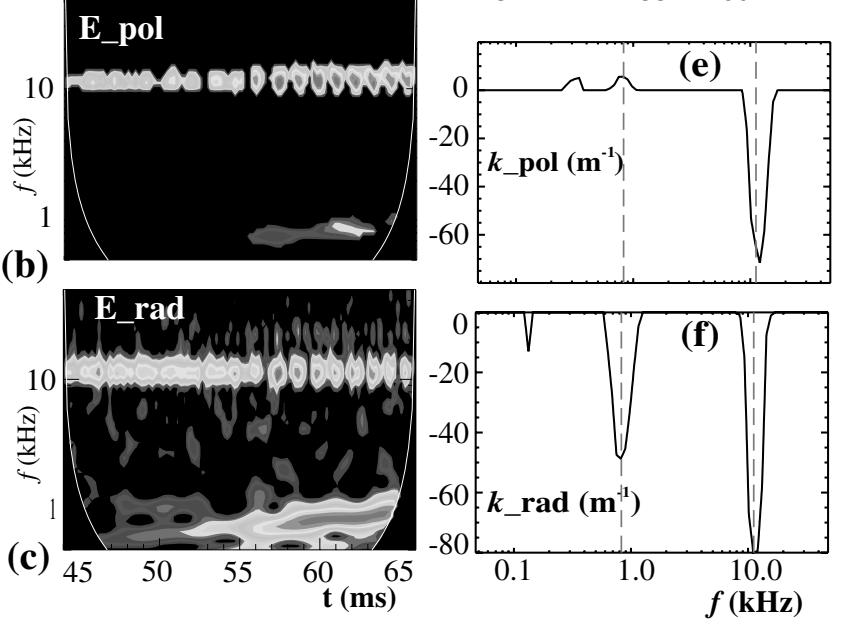

FIG. 3. Time-frequency intensity evolution of the fluctuations in the radial (a) and poloidal (b) electric fields, density fluctuations (c), and the time evolution of the integrated fluctuation-driven particle flux (d) at $r / a=0.51$ during the $H$-mode discharge. Also shown are time-integrated frequency spectra of the poloidal (e) and radial (f) wave numbers of the plasma potential fluctuations.

poloidal electric field [Fig. 3(b)], and radial electric field [Fig. 3(c)]. Later this harmonic broadens, exhibiting two peaks at $f=11.0 \mathrm{kHz}$ and $f=11.95 \mathrm{kHz}$. Higher harmonics at $f=22.1 \mathrm{kHz}$ and $f=23.0 \mathrm{kHz}$ also develop after $t \sim 54 \mathrm{~ms}$. This broadening is seen in the wavelet spectra of Figs. 3(a)-3(c). The individual frequencies cannot be resolved by wavelets (frequency resolution is traded off in favor of time resolution), but can be seen in the Fourier spectra. Broadening in the fluctuation spectra coincides with the growth of the low-frequency feature at $F_{r} \approx 0.9 \mathrm{kHz}$, which is first observed in the $\tilde{E}_{r}$ spectrum at $t \sim 54 \mathrm{~ms}$ and then later in the $\tilde{n}_{e}$ and $\tilde{E}_{\theta}$ fluctuation spectra. The development of this $F_{r} \approx 0.9 \mathrm{kHz}$ activity leads to a low-frequency modulation of the higherfrequency harmonics of all fluctuations seen after $t \sim$ $55 \mathrm{~ms}$ in Figs. 3(a)-3(c). This also leads to the modulation in the fluctuation-driven particle flux driven by these modes as shown in Fig. 3(d). The flux is computed as discussed in [28]. As seen in Figs. 3(e) and 3(f), the $f=11 \mathrm{kHz}$ fluctuations have comparable poloidal and radial wave numbers, $k_{\theta}(f) \sim 70 \mathrm{~m}^{-1}, k_{r}(f) \sim 80 \mathrm{~m}^{-1}$, while the low-frequency component has the radial wave number $k_{r}\left(F_{r}\right) \sim 50 \mathrm{~m}^{-1}$ which is much larger than the poloidal one $\left[k_{\theta}\left(F_{r}\right) \sim 5 \mathrm{~m}^{-1}\right]$. Figure 4 shows the cross bicoherence and the summed cross bicoherence computed for two time intervals of the discharge illustrated in Fig. 3. A relatively low nonlinear coupling between $f=11 \mathrm{kHz}$ 


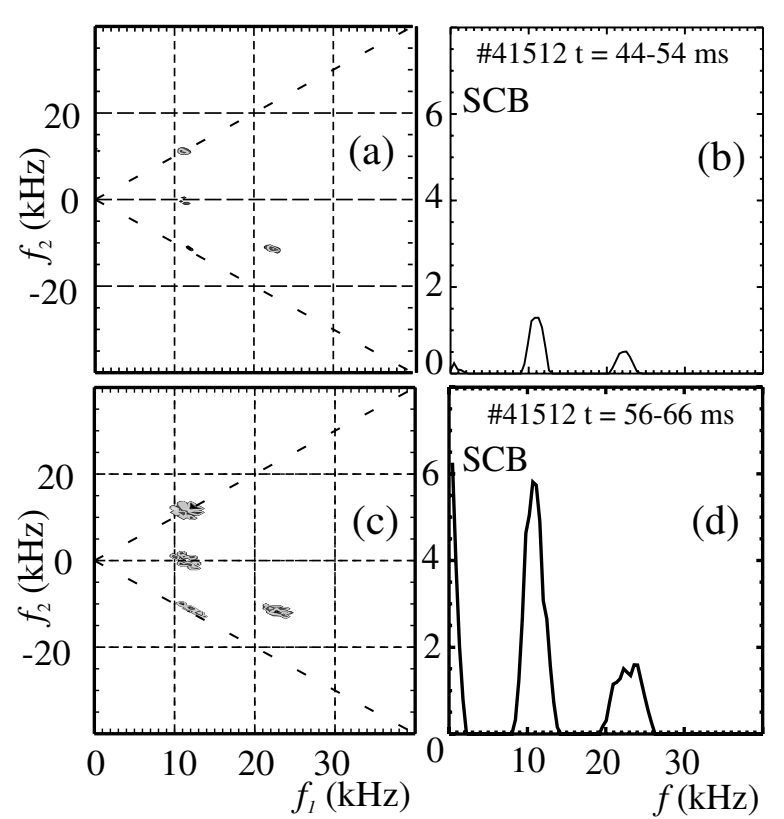

FIG. 4. Contour plots of the cross bicoherence and plots of the summed cross bicoherence computed for two time intervals of the discharge shown in Fig. 3. Panels (a) and (b) correspond to $t=44-54 \mathrm{~ms}$, while (c) and (d) correspond to $t=56-66 \mathrm{~ms}$.

and $f=22 \mathrm{kHz}$ components is seen at $t=44-54 \mathrm{~ms}$ [Figs. 4(a) and 4(b)]. Later in time $(t=56-66 \mathrm{~ms})$ the level of nonlinear coupling to all frequency components is significantly increased, as shown in Figs. 4(c) and 4(d). The SCB in Fig. 4(d) shows that the low-frequency component at $F_{r} \approx 0.9 \mathrm{kHz}$ has the strongest coupling to other modes. Summarizing these observations, during the development of the nonlinear fluctuations in $H$ mode a broadening of the fluctuation frequency spectra coincides with the large increase in the degree of nonlinear coupling between modes. This increased coupling correlates with the formation of the low-frequency $\left(F_{r} \approx 0.9 \mathrm{kHz}\right)$ potential structure whose radial wave number is much larger than the poloidal one. The onset of this structure leads to the strong amplitude modulation of the higher-frequency harmonics in all fluctuating quantities, including the fluctuation-driven particle flux.

In summary, we report for the first time direct measurements of the poloidally symmetric radially localized $\left(k_{r} \gg k_{\theta} \approx 0\right)$ potential structures formed as a result of 3 -wave interactions with the higher $k_{\theta}$ modes. Presented results are qualitatively consistent with the theoretical picture of the zonal flow generation and self-regulation of turbulence by zonal flows [4]. Strong low-frequency components in the radial electric field in the $\mathrm{H}-1$ heliac are observed in both low and high confinement modes. Fluctuations in these two modes are believed to have different energy reservoirs: a pressure gradient in $L$ mode and the radial electric field shear in $H$ mode (Kelvin-Helmholz instability). Nevertheless, the formation of the $\tilde{\mathbf{E}} \times \mathbf{B}$ flows is observed in both modes, suggesting the universality of the mechanism. The flow interaction with the higher $k_{\theta}$ fluctuations is similar to that between zonal flows and their parent drift waves including the nonlinear coupling between them, as reflected in the bispectra. Such coupling is observed only after the unstable wave spectrum develops a sufficient number of nonlinearly coupled harmonics, as shown in Fig. 4. The generation of the $\tilde{\mathbf{E}} \times \mathbf{B}$ flows in our experiments correlates with the remarkable modification in the fluctuation-driven particle fluxes [Fig. 3(d)]. Although we do not insist here on the exact analogy between the observed potential structures and zonal flows, it is not impossible that the fluctuation self-organization in our experiments is based on a qualitatively similar physics principle.

We are grateful to P. H. Diamond, T. S. Hahm, and M. A. Malkov for useful discussions.

*Electronic address: Michael.Shats@anu.edu.au

[1] K. Itoh and S. I. Itoh, Plasma Phys. Controlled Fusion 38, 1 (1996).

[2] P. W. Terry, Rev. Mod. Phys. 72, 109 (2000).

[3] A. Hasegawa and M. Wakatani, Phys. Rev. Lett. 59, 1581 (1987).

[4] P. H. Diamond et al., Proceedings of the 17th IAEA Fusion Energy Conference, Yokohama, Japan, 1998 (IAEA, Vienna, 1998), p. IAEA-CN-69/TH3/1.

[5] A. M. Balk et al., Sov. Phys. JETP 71, 249 (1990).

[6] A. I. Smolyakov et al., Phys. Rev. Lett. 84, 491 (2000).

[7] M. A. Malkov et al., Phys. Plasmas 8, 5073 (2001).

[8] Z. Lin et al., Science 281, 1835 (1998).

[9] A. Dimits et al., Phys. Rev. Lett. 77, 71 (1996).

[10] K. Hallatschek and D. Biskamp, Phys. Rev. Lett. 86, 1223 (2001).

[11] T. S. Hahm et al., Plasma Phys. Controlled Fusion 42, A205 (2000).

[12] P. H. Diamond et al., Phys. Rev. Lett. 84, 4842 (2000).

[13] S. Coda, M. Porkolab, and K. H. Burrel, Phys. Rev. Lett. 86, 4835 (2001).

[14] G. R. Tynan et al., Phys. Plasmas 8, 2691 (2001).

[15] R. A. Moyer et al., Phys. Rev. Lett. 87, 135001 (2001).

[16] P.H. Diamond and Y.B. Kim, Phys. Fluids B 3, 1626 (1991).

[17] F. L. Hinton and M. N. Rosenbluth, Plasma Phys. Controlled Fusion 41, A653 (1999).

[18] W. M. Solomon and M. G. Shats, Phys. Rev. Lett. 87, 195003 (2001).

[19] S. M. Hamberger et al., Fusion Technol. 17, 123 (1990).

[20] M. G. Shats et al., Phys. Plasmas 4, 3629 (1997).

[21] S. L. Chen and T. Sekiguchi, J. Appl. Phys. 36, 2363 (1965).

[22] M. G. Shats, Plasma Phys. Controlled Fusion 41, 1357 (1999).

[23] D. L. Rudakov et al., Plasma Phys. Controlled Fusion 43, 559 (2001).

[24] M. G. Shats and D. L. Rudakov, Phys. Rev. Lett. 79, 2690 (1997).

[25] C. Torrence and G. P. Compo, Bull. Am. Meteorol. Soc. 79, 61 (1998).

[26] B. Ph. Van Milligen et al., Phys. Plasmas 2, 3017 (1995).

[27] V. Dose et al., Phys. Plasmas 4, 323 (1997).

[28] X. Shi et al., Rev. Sci. Instrum. 72, 503 (2001). 